Int. J. Electrochem. Sci., 16 (2021) Article ID: 210812

International Journal of

ELECTROCHEMICAL

SCIENCE

www.electrochemsci.org

\title{
Monitoring Glucose in Fermented Beer by an Electrochemical Sensor Based on Graphene Oxide Decorated by Silver Nanoparticles
}

\author{
Yin Feng ${ }^{*}$, Yan Liu* ${ }^{*}$ Bo Feng, Haiyan Chen, Lixin You ${ }^{1}$, Huaiquan Pei ${ }^{1}$ \\ School of Life Sciences, Changchun SCI-TECH University, Changchun 130000, China \\ *E-mail: fengyin1123@sina.com, 1y78210310@sina.com
}

doi: $10.20964 / 2021.08 .14$

Received: 7 April 2021/ Accepted: 26 May 2021 / Published: 30 June 2021

\begin{abstract}
The aim of this research was to create a non-enzymatic sensor for electrochemical glucose monitoring during the glycolytic process using functionalized graphene oxide decorated with silver nanoparticles modified glassy carbon electrode (Ag NPs/f-GOx/GCE). The Hummers method was used to synthesize GOx, which was then functionalized before being decorated with Ag NPs. The prepared GOx nanosheets oriented at different angles, rippled, and entangled with each other, and the Ag NPs in fcc structure were strongly and irregularly immobilized on GOx nanosheets in spherical form, according to morphological and structural studies using SEM and XRD analyses. The electrochemical studies using CV and amperometry techniques revealed that Ag NPs/f-GOx/GCE has a high stability and sensitivity, with sensitivity, detection limit, and linear range of $2.94 \mu \mathrm{A} / \mu \mathrm{M}, 0.001 \mu \mathrm{M}$, and 0 to $28 \mu \mathrm{M}$, respectively. When comparing the glucose sensing properties of Ag NPs/f-GOx/GCE to those of other non-enzymatic glucose sensors, it was discovered that Ag NPs/f-GOx/GCE had the lowest detection limit and the highest sensitivity values due to the synergetic effect of Ag NPs and f-GOx on Ag NPs/f-GOx/GCE, which have great structural characteristics and significant amounts of electroactive sites. The analysis of interference effect in the presence of potential interfering species during the glycolytic process revealed the prepared glucose sensor's high sensitivity and selectivity. The practical application of the proposed sensor to determine glucose in a fermented beer sample revealed that Ag NPs/f-GOx/GCE has the potential to be a reliable glucose determination sensor during glucose fermentation.
\end{abstract}

Keywords: Glucose; Amperometry; fermentation; Graphene oxide; Silver nanoparticles

\section{FULL TEXT}

(C) 2021 The Authors. Published by ESG (www.electrochemsci.org). This article is an open access article distributed under the terms and conditions of the Creative Commons Attribution license (http://creativecommons.org/licenses/by/4.0/). 